Bundesgesundheitsbl -

Gesundheitsforsch - Gesundheitsschutz

2000 • 43:264-271 @ Springer-Verlag 2000

Originalien und Übersichtsarbeiten

I. Schöneberg • Robert Koch-Institut, Berlin

\title{
Erkrankungen an Paratyphus in Deutschland
}

\section{Daten aus Einzelfallerhebungen des Robert Koch-Institutes unter besonderer Berücksichtigung einer Erkrankungshäufung im Sommer 1999}

\section{Zusammenfassung}

Die Anzahl der Erkrankungen an Paratyphus in Deutschland hat sich in den letzten Jahren - im Vergleich zu früher - verringert. In den Vorjahren (seit 1997) wurden in jedem Jahr ca. 60 Erkrankungen gemeldet, die zu einem großen Teil aus anderen Ländern importiert wurden. Im Sommer 1999 erhöhte sich die Zahl der erfassten Erkrankungen durch einen Ausbruch von Paratyphus B nach Türkeireisen. In neun verschiedenen Ländern Europas kam es zu insgesamt mehr als 300 Erkrankungen an Paratyphus B. In Deutschland wurden in diesem Zusammenhang $40 \mathrm{Er}$ krankungsfälle registriert. Die Laboruntersuchungen ergaben, dass alle untersuchten Paratyphus B-Stämme den Lysotyp Taunton/B 7 aufwiesen.

\section{Schlüsselwörter}

Salmonella Paratyphi · Zahl der Fälle · Ausbruch · Import von Fällen · Laboruntersuchungen
Paratyphus gehört zu den Erkrankungen, die in früheren Jahrzehnten in Deutschland in wesentlich größerer Häufigkeit auftraten, heute jedoch nur in geringen Fallzahlen registriert werden.

Gute hygienische Bedingungen sowie eine effektive Antibiotikatherapie führten dazu, dass Erkrankungen an Paratyphus heute in der Regel in Deutschland kein besonderes gesundheitspolitisches Problem mehr darstellen, obwohl es bei den einzelnen Erkrankungen durchaus zu schwereren Verläufen, sehr selten auch zu Todesfällen kommen kann.

$\mathrm{Da}$ in vielen anderen Ländern - vor allem in Entwicklungsländern - die Situation grundsätzlich anders ist und $\mathrm{Pa}$ ratyphus dort noch zum Teil weit verbreitet vorkommt $[1,2]$, werden durch Reisen und damit verbundene vielfältige Kontakte immer wieder ParatyphusErkrankungen nach Deutschland eingeschleppt.

Die Erreger des Paratyphus werden durch kontaminiertes Wasser oder Lebensmittel auf den Menschen übertragen. Unerkannte Ausscheider können den Erreger über Monate bzw. sogar Jahre tragen und somit eine Infektionsquelle für andere Menschen darstellen. Daher kommt der Erfassung von Ausscheidern sowie einer Verhinderung von Tätigkeiten dieses Personenkreises in der Lebensmittelherstellung eine wichtige Rolle zu [3].
Aufgrund der unterschiedlichen Antigenstruktur werden im KauffmannWhite-Schema Salmonella Paratyphi A, $B$ und $C$ unterschieden. Anhand der unterschiedlichen Fermentation von dTartrat werden die d-Tartrat-positiven Stämme als Varietät S. Java von den "echten" S. Paratyphi B-Stämmen abgegrenzt, die stets d-Tartrat-negativ sind. Mittels Lysotypie können S. Paratyphi A- und BStämme zur epidemiologischen Aufklärung von Infektketten weiter unterteilt werden.

\section{"Die Erreger des Paratyphus werden durch kontaminiertes Wasser oder Lebensmittel auf den Menschen übertragen."}

Während S. Paratyphi C ausschließlich beim Menschen vorkommt, ist S. Paratyphi B auch bei Tieren (Rind, Schwein, Hausgeflügel $u$. a.) und in der Umwelt (See- und Abwasser) verbreitet. Allerdings ist meist nicht angegeben, ob die dTartrat-negative ("echte" S. Paratyphi B) oder d-Tartrat-positive (S. Java) Variante nachgewiesen wurde, so dass es sich nahezu ausschließlich um S. Java handeln dürfte. Auch S. Paratyphi A wird gelegent-

Dr. Irene Schöneberg Robert Koch-Institut, Postfach 6502 80, 13302 Berlin 


\section{I.Schöneberg}

Cases of Paratyphoid Fever in Germany. Data from single case investigation of the Robert Koch-Institute with special respect of an outbreak episode in summer 1999

\section{Summary}

The number of infections with Salmonella Paratyphi has decreased in recent years in Germany. Since 1997 about 60 cases were reported annually. Most of the cases were imported from other countries. In 1999 the number of reported cases increased: an outbreak of S. Paratyphi B associated with travel to Turkey was recognized. More than 300 cases of infections with S. Paratyphi B were identified in 9 different European countries. Fourty of the cases were detected in Germany. The laboratory investigations showed that all strains belonged to the phage type Taunton/B7.

\section{Key words}

Salmonella Paratyphi · Number of Cases . Outbreak · Importation of Cases - Laboratory Investigation lich bei Hausgeflügel isoliert [4]. S. Paratyphi A, B und C verursachen die septikämischen Allgemeininfektionen Paratyphus A, B und C. Alle S. Paratyphus-Infektionen können auch als Gastroenteritis verlaufen. Das gilt nahezu immer für Infektionen durch S. Java, die d-Tartratpositive Varietät von S. Paratyphi B.

\section{Meldepflicht nach Bundes-Seuchengesetz}

Paratyphus ist nach Bundes-Seuchengesetz (BSeuchG) meldepflichtig. Ärzte in Krankenhäusern, ambulant tätige Ärzte oder Ärzte aus privaten und öffentlichen Untersuchungsstellen (Untersuchungsämter, Institute, Laboratorien) melden den Krankheitsverdacht, die Erkrankung, den Tod an Paratyphus A, B und C sowie die Ausscheider von $S$. Paratyphi $A, B$ und $C$ dem - nach dem Wohnsitz des Erkrankten - örtlich zuständigen Gesundheitsamt ( $\$ \$_{3}, 4,5,9$ BSeuchG). Darüber hinaus ist in $\$ 48$ BSeuch G eine spezielle Meldepflicht festgelegt, die für Leiter von Einrichtungen zur Unterbringung und Betreuung von Kindern gilt.

Über die Bezirksregierungen bzw. Behörden der Bundesländer gelangt die Meldung der Erkrankung bzw. des Todesfalles zum Robert Koch-Institut (RKI). Entsprechend BSeuch G werden den Gesundheitsämtern die Erkrankungen an Paratyphus, differenziert nach A, B oder $C$ gemeldet. Die Weitergabe der Daten an das RKI erfolgt jedoch aus den meisten Ländern nicht unterteilt nach Paratyphus A, B und C, d.h. die im RKI erfassten Meldedaten gestatten keine Unterscheidung in Paratyphus A, B oder C.
Für Erkrankungen an Paratyphus liegt im RKI ein spezieller Erhebungsbogen vor. Dieser wird im Falle einer Meldung von Erkrankungen an Paratyphus an die zuständigen Gesundheitsämter bzw. Landesbehörden gegeben. Im engen Zusammenwirken mit den örtlichen Gesundheitsämtern wird anhand des Bogens versucht, Angaben und Daten für jeden gemeldeten Einzelfall zu erhalten.

Im folgenden wird über das Auftreten von Erkrankungen und Sterbefällen an Paratyphus in den letzten Jahrzehnten in Deutschland berichtet. Dabei soll eine Häufung von Paratyphus B-Erkrankungen in Deutschland und in acht weiteren europäischen Ländern nach Reisen in die Türkei im Sommer 1999 besondere Berücksichtigung finden.

\section{Zahl der in Deutschland erfassten Erkrankungen an Paratyphus}

In den vergangenen Jahrzehnten hat die Bedeutung von Erkrankungen an Paratyphus in Deutschland ständig abgenommen $[3,5,6]$. Insbesondere nach dem 2. Weltkrieg wurden in Deutschland jährlich mehrere Tausend Erkrankungsfälle erfasst (Tabelle 1). Gegenwärtig zählen Erkrankungen an Paratyphus zu den eher selten auftretenden Infektionskrankheiten, die zudem zu einem gröBeren Teil aus dem Ausland nach Deutschland importiert werden. In den letzten Jahren (seit 1996) wurden in Deutschland jährlich deutlich unter 100 Erkrankungsfälle an Paratyphus gemel$\operatorname{det}($ Abb. 1).

\begin{tabular}{|c|c|c|c|}
\hline Jahr & $\begin{array}{l}\text { Alte Länder } \\
\text { (früheres Bundesgebiet) }\end{array}$ & $\begin{array}{l}\text { Neue Länder } \\
\text { (frühere DDR) }\end{array}$ & $\begin{array}{l}\text { Deutschland } \\
\text { (gesamt) }\end{array}$ \\
\hline 1950 & 5704 & 1479 & 7183 \\
\hline 1960 & 2406 & 244 & 2650 \\
\hline 1970 & 540 & 98 & 638 \\
\hline 1980 & 212 & 15 & 227 \\
\hline 1990 & 126 & 3 & 129 \\
\hline 1998 & 55 & 6 & 61 \\
\hline
\end{tabular}

Zahlen nach $[5,6]$ 


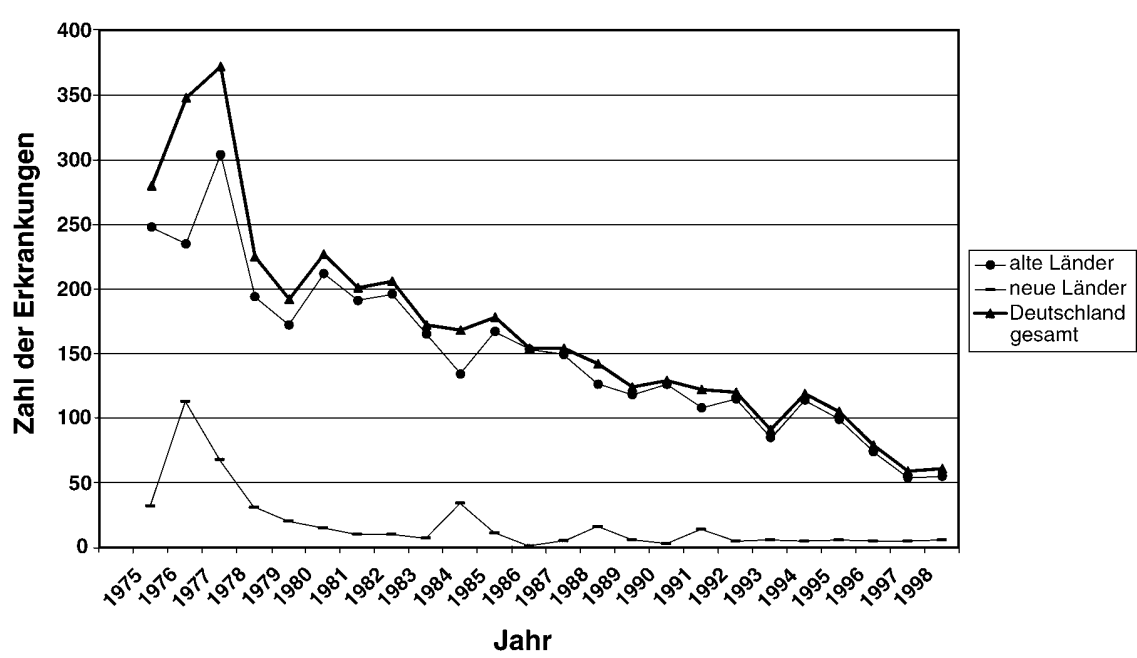

Abb. $1 \Delta$ Paratyphus in Deutschland - gemeldete Erkrankungen 1975 bis 1998
Daten zur Morbidität, d.h. der Zahl der erfassten Erkrankungen pro 100000 Einwohner, machen den Rückgang der Erkrankungszahlen in Deutschland besonders deutlich. So wurden noch 1950 10,5 Erkrankungen pro 100 ooo Einwohner registriert, im Jahre 1997 waren es 0,072 Erkrankungen pro 100 ooo Einwohner.

\section{„Nach dem 2.Weltkrieg wurden in Deutschland jährlich mehrere Tausend Paratyphusfälle erfasst. In den letzten Jahren lagen die Zahlen deutlich unter hundert."}

Da es sich bei den Paratyphus-Erkrankungen zu einem beträchtlichen Teil um importierte Fälle handelt, wurden auch in den Vorjahren entsprechend mehr Erkrankungen im 3. Quartal - dem Zeitraum der Hauptreisezeit im Sommer gemeldet. 1999 kam es - bedingt durch die Häufung an Paratyphus B-Fällen aus der Türkei - zu einem deutlichen Anstieg der erfassten Paratyphus B-Erkrankungen im 3. Quartal (Abb. 2).

Angaben zu Sterbefällen an Paratyphus liegen in Deutschland durch die Todesursachenstatistik des Statistischen Bundesamtes vor [5, 7]. In den Nachkriegsjahren, den Jahren mit den größten Erkrankungszahlen, wurden in Deutschland auch in jedem Jahr eine Reihe von Sterbefällen infolge Paratyphus registriert (1949: 107; 1950: 126).
In den letzten Jahren (seit 1994) wurden durch die Todesursachenstatistik des Statistischen Bundesamtes [7] keine Todesfälle infolge Paratyphus ausgewiesen. Lediglich in den Jahren 1988 und 1993 war je ein Sterbefall zu verzeichnen.

\section{Einzelfallerhebungen des RKI 1997/98}

Einzelfalldaten sind im RKI für 80 bis 90\% aller Erkrankungen an Paratyphus seit 1997 vorhanden. In den Vorjahren war die wahrscheinliche Infektionsquelle für eine Vielzahl der Fälle im Ausland (1997: 65\%, 1998: 54\%). Besonders häufig kamen die importierten Erkrankungen aus Ländern Asiens (Abb.3). So traten eine Reihe von Erkrankungen nach
Reisen in Indien (acht), Pakistan (acht) oder Indonesien (drei) auf. Auch in der Türkei wurden Paratyphus-Infektionen in den vergangenen Jahren in vielen Fällen akquiriert. Während im Jahre 199713 der insgesamt 51 über Sondererhebungen erfassten Paratyphus-Erkrankungen in der Türkei erworben wurden, waren es 1998 acht von 54 Fällen. Anzumerken ist jedoch in diesem Zusammenhang, dass die in den Vorjahren aus der Türkei nach Deutschland importierten Erkrankungsfälle an Paratyphus zu einem großen Teil auf Bürger türkischer Herkunft zurückzuführen waren (1997: elf von 13, 1998: sechs von acht). Unter den 1997/98 in Deutschland an Paratyphus Erkrankten befand sich ebenfalls eine größere Zahl von Bürgern anderer Länder. So wurden ca. $45 \%$ der erfassten Fälle bei Patienten ausländischer Herkunft festgestellt. Entsprechend den Angaben aus den Einzelfallerhebungen wurden 1997/98 insgesamt 33 bzw. 29 Erkrankungen an Paratyphus nach Deutschland importiert. Deutsche akquirierten ihre Infektion in zwölf bzw. elf Fällen durch Auslandsreisen.

Wie schon beschrieben, sind die nach BSeuchG im RKI erfassten Meldedaten nicht nach Paratyphus A, B oder C differenziert. Da in den Einzelfalldaten auch die Ergebnisse der durchgeführten diagnostischen Untersuchungen enthalten sind, ermöglichte eine Analyse der Sondererhebungen auch Angaben zu den Erregertypen. Es zeigte sich, dass annähernd die Hälfte (1997: 49\%; 1998: $48 \%)$ der in Deutschland gemeldeten Erkrankungen an Paratyphus durch $S$.

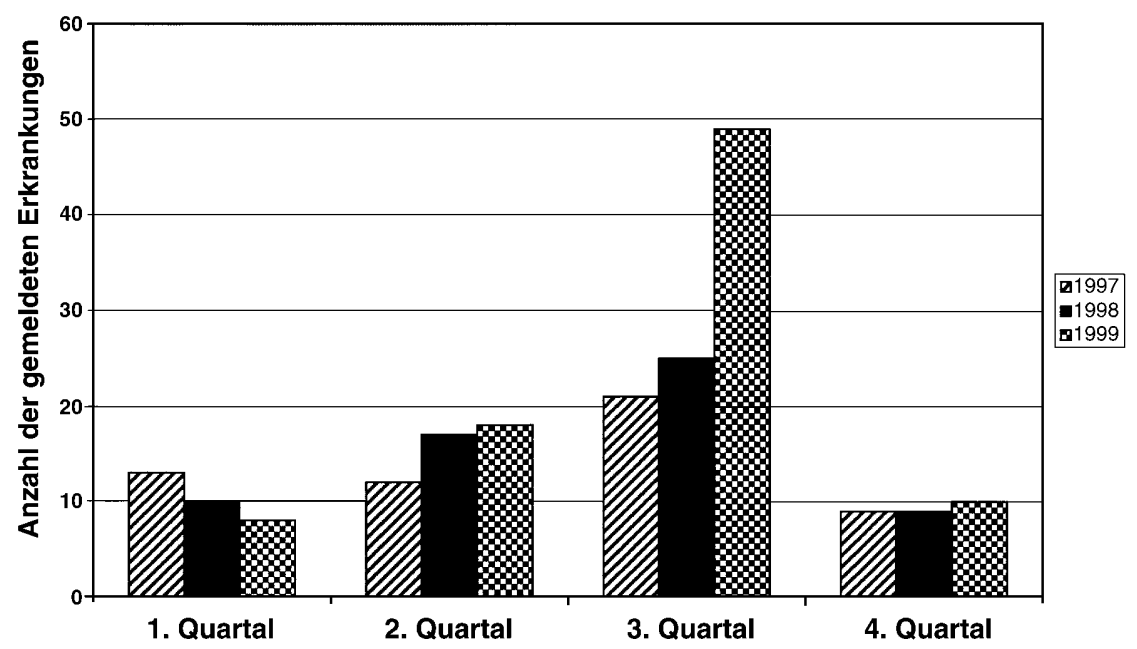

Abb. 2 Paratyphus in Deutschland - gemeldete Erkrankungen nach Quartalen 


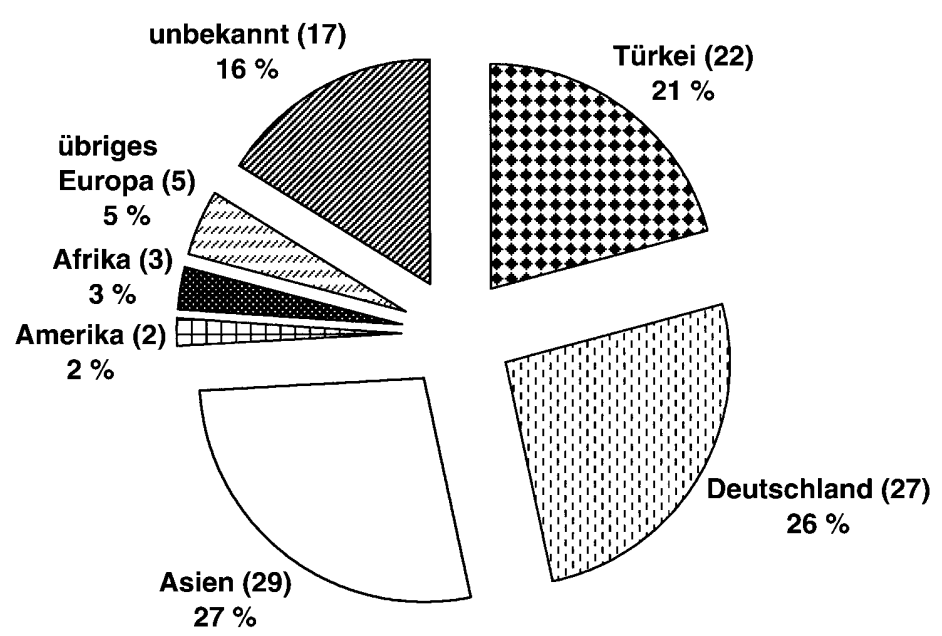

Abb. $3 \triangle$ Paratyphus in Deutschland 1997/98 - wahrscheinliches Infektionsgebiet

Paratyphi $B$ verursacht wurde (Abb. 4). Erkrankungen durch S. Paratyphi A lagen mit 25 bzw. mit 33\% an zweiter Stelle nach Häufigkeit, gefolgt von S. Paratyphi C (1997: 6\%; 1998: 4\%). Für 20 bzw. $15 \%$ der Fälle erfolgte keine nähere Angabe zum Erregertyp. Insgesamt kamen nur fünf Fälle, die durch S. Paratyphi C verursacht worden waren, zur Meldung. Diese betrafen in drei Fällen Patienten nach Reisen (Asien, Polen, Spanien). Für zwei ältere Patienten (79 bzw. 74 Jahre alt) fehlten Anhaltspunkte für eine Infektionsquelle völlig.

In den Jahren 1997/98 waren Personen verschiedener Altersgruppen von Erkrankungen an Paratyphus betroffen (Abb. 5). Insgesamt waren Kinder und jüngere Erwachsene an den ParatyphusErkrankungen jedoch häufiger beteiligt als ältere Erwachsene. Erkrankungen wurden aber auch bei Patienten sehr hohen Alters beobachtet. Die ältesten in den Sondererhebungen erfassten, an Paratyphus erkrankten Personen waren 87 bzw. 91 Jahre alt. Zirka 2/3 aller in Deutschland an Paratyphus Erkrankten wurden stationär im Krankenhaus behandelt (1997: 65\%; 1998: 67\%).

\section{Ausbruch von Paratyphus B-Erkrankungen nach Türkeireisen im Sommer 1999}

Nachdem in verschiedenen nordeuropäischen Ländern (Norwegen, Dänemark, Schweden, England und Wales, Finnland) schon eine größere Zahl von Erkrankungen an Paratyphus $B$ nach Türkeireisen (Region Alanya und Antalya) registriert worden war (siehe weite- re Ausführungen), war davon auszugehen, dass auch in Deutschland, dem Land mit der wohl größten Zahl von Reisenden in die Türkei, gehäuft Erkrankungen auftreten müssten. $\mathrm{Zu}$ diesem Zeitpunkt waren europaweit insgesamt 109 Erkrankungen an Paratyphus B bekannt, davon sechs in Deutschland [8]. Seitens der anderen europäischen Länder wurde das RKI aufgefordert, eine aktive Fallfindung für Paratyphus B-Erkrankungen, die nach Türkeireisen aufgetreten waren, in Deutschland zu initiieren. Dabei galt folgende vorläufige Falldefinition: Personen, bei denen $S$. Paratyphi $B$ isoliert wurde, die in der Türkei gewesen waren und deren Erkrankung nach dem 30.6.1999 begonnen hatte, sollten diesem Ausbruch zugerechnet werden.

\section{"Nachdem im Sommer 1999 vermehrt über Paratyphusfälle bei Türkei-Reisenden berichtet wurde, ist in Deutschland vom RKI eine aktive Fallfindung initiiert worden."}

Über die obersten Landesgesundheitsbehörden - und gleichzeitig durch das "Epidemiologische Bulletin" - wurden alle Gesundheitsämter in Deutschland gebeten, vor allem die Krankenhäuser und Laboratorien in die aktiven Ermittlungen einzubeziehen. Die Erfassung der Angaben zu den ermittelten Fällen erfolgte im RKI, das aus den meisten Bundesländern alle Informationen in direktem Kontakt mit den örtlichen Gesundheitsämtern erhielt. Die unmittelbare Zusammenarbeit von Gesundheitsämtern und RKI konnte in den meisten Bundesländern mit Zustimmung der Obersten Landesbehörden erfolgen. So gelang es, den Meldeweg für diese speziellen Fälle wesentlich zu beschleunigen. Aufgrund des zeitnahen Bekanntwerdens der Fälle wurde es im Rahmen dieser Aktion möglich, die Weitergabe des größten Teiles aller in diesem Zusammenhang in den bakteriologischen Laboratorien vorliegenden Stämme an das Nationale Referenzzentrum (NRZ) für Salmonellen und andere Enteritiserreger am RKI, Bereich Wernigerode zur Feintypisierung zu veranlassen.

Im Zeitraum der aktiven Fallfindung wurden insgesamt 43 Fälle an Pa-

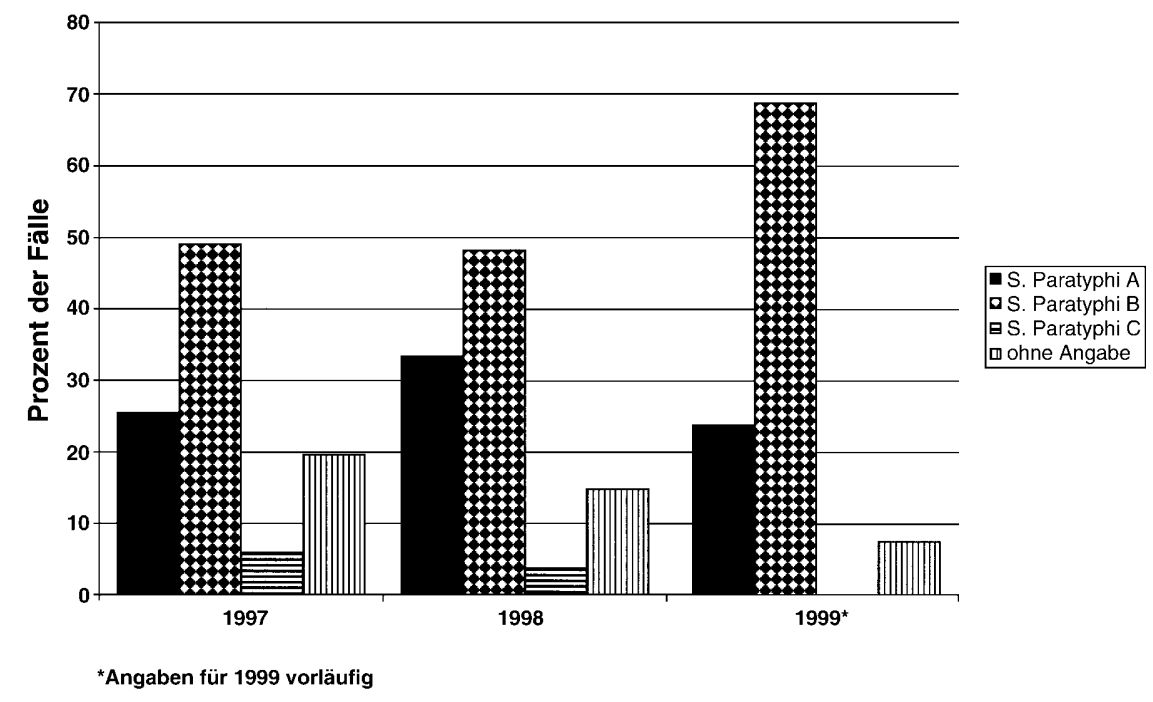

Abb. $4 \Delta$ Paratyphus in Deutschland - Erregertyp 
ratyphus B bekannt, die in Deutschland nach Reisen in die Türkei aufgetreten waren. Nicht alle Fälle wurden in direktem Zusammenhang mit der aktiven Fallsuche eruiert - ein Teil der Fälle wurde auch über die routinemäßigen Wochenmeldungen an das RKI gegeben. Ein anderer Teil der Fälle wurde durch Aktivitäten bakteriologischer Laboratorien ermittelt, d.h. durch Übermittlung des Ergebnisses der Feintypisierung im NRZ und nachfolgender Zuordnung des Befundes zu aufgetretenen Fällen mit Hilfe der für den Wohnort des Betroffenen zuständigen Gesundheitsämter. Da alle Fälle letztendlich über die örtlichen Gesundheitsämter erfasst wurden, konnten Doppelmeldungen bereits auf der Ebene der Gesundheitsämter ausgeschlossen werden.

Im Rahmen dieses Erkrankungsgeschehens wurden in Deutschland im einzelnen 40 Erkrankungsfälle und drei asymptomatische Ausscheider registriert. Weiterhin wurden im Laufe der Erhebungen die Erkrankungen zweier Niederländer bekannt, die ebenfalls im entsprechenden Zeitraum in der Türkei geweilt hatten, in der Folge an Paratyphus $B$ erkrankt waren und in einer deutschen Klinik stationär behandelt worden waren. In Deutschland kam es im Laufe des Erkrankungsgeschehens zu zwei Kontakterkrankungen an Paratyphus B, die auf bekannte, aus der Türkei importierte Erkrankungen zurückgingen. Für alle in diesem Zusammenhang bekannten Fälle wurde der Zeitpunkt der Erkrankung mit Mitte Juli bis Anfang September angegeben (Beginn der ersten Erkrankung: 17.7.1999; Beginn der letzten Erkrankung: 1.9.1999).

Die während des Ausbruches aus der Türkei importierten Paratyphus BErkrankungen kamen fast ausschließlich bei Kindern älterer Jahrgänge $(>5$ Jahre) und bei jungen Erwachsenen $(<25$ Jahre) vor (Abb. 6). Nur in einzelnen Fällen (insgesamt sechs Erkrankte) waren Erwachsene anderer Altersgruppen betroffen. Die älteste in diesem $\mathrm{Zu}$ sammenhang erfasste Patientin, die Mutter eines an Paratyphus B erkrankten 22jährigen Mannes, war 45 Jahre alt und türkischer Nationalität. Neben diesen Fällen, die innerhalb einer Familie auftraten und Mutter und Sohn betrafen, wurden zwei weitere im Zusammenhang stehende Infektionen bekannt. Die mitreisende Freundin eines erkrankten

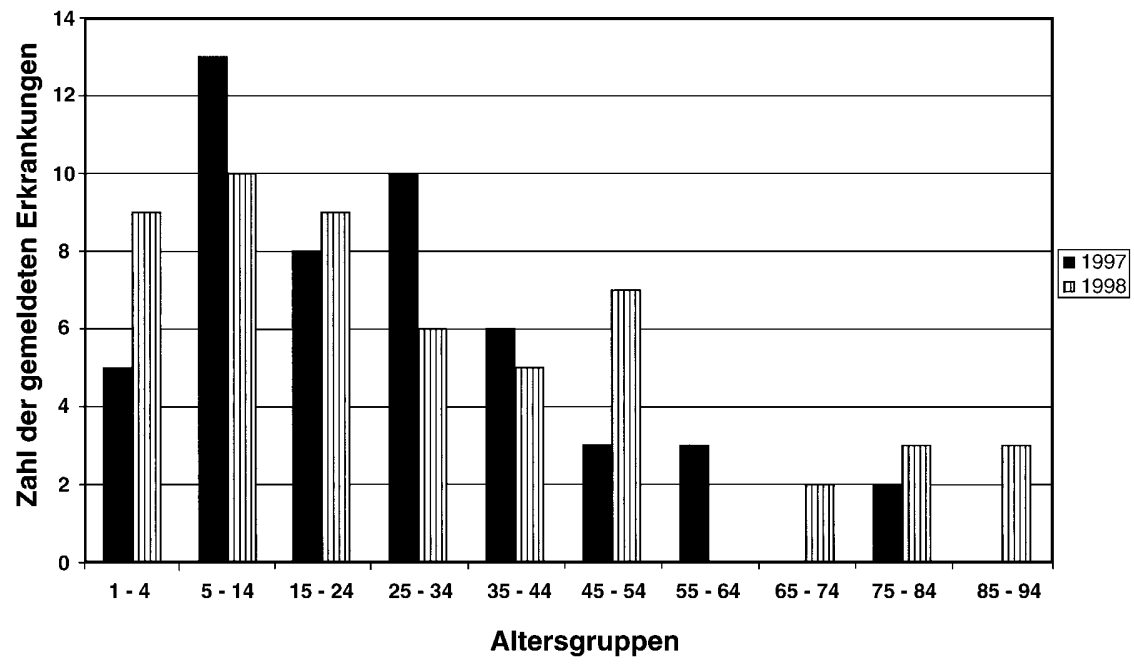

Abb. $5 \Delta$ Paratyphus in Deutschland - Altersgruppen der gemeldeten Erkrankungen

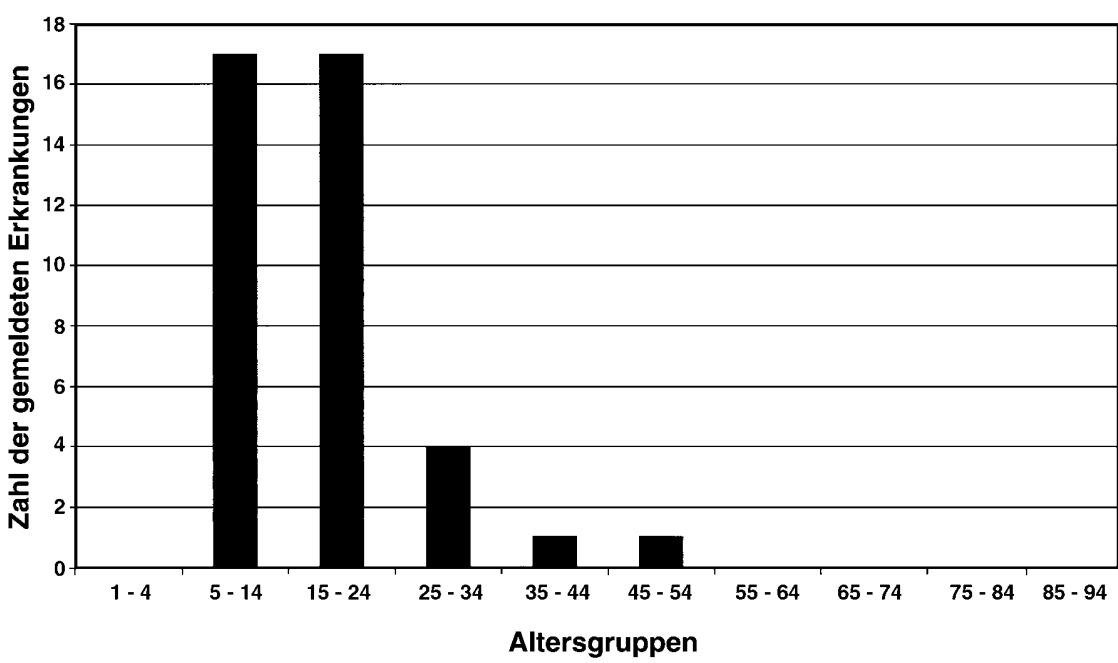

Abb.6 $\triangle$ Häufung an Paratyphus B-Erkrankungen 1999 - Altersgruppen der gemeldeten Erkrankungen

jungen Mannes wurde als Ausscheiderin von S. Paratyphi $B$ ermittelt. Von den Erkrankungen an Paratyphus B waren männliche und weibliche Personen nahezu gleichermaßen betroffen (18 weibliche, 22 männliche Personen). Die klinische Symptomatik bei den Erkrankten war unterschiedlich ausgeprägt. Für einen größeren Teil der Patienten wurde Fieber und Durchfall, teilweise mit Kopfschmerzen, angegeben. 29 der $40 \mathrm{Er}$ krankten waren hospitalisiert worden (entsprechend $72 \%$ ). Todesfälle waren unter den nach Türkeireisen im Sommer 1999 an Paratyphus B Erkrankten nicht zu beklagen. Bei den im Rahmen des hier beschriebenen Paratyphus B-Ausbruches erfassten Erkrankungen waren türkische Bürger in elf Fällen (von 40) beteiligt. Dagegen betrafen 29 der aufge- tretenen Erkrankungen sowie die drei ermittelten Ausscheider Bürger deutscher Nationalität. Paratyphus B-Fälle nach Türkeiaufenthalten im Sommer 1999 wurden in Deutschland aus insgesamt zwölf Bundesländern bekannt (Tabelle 2).

Neben den Erkrankungen an Paratyphus B, die Gegenstand dieser Betrachtungen sind, traten im gleichen Zeitraum nach Türkeireisen auch Erkrankungen an Paratyphus A (vier Fälle) und an Typhus (15 Fälle) auf.

\section{Differenzierung und Feintypisierung der Stämme}

Die Identifizierung und Differenzierung der S. Paratyphi B-sowie S. Paratyphi Aund $C$-Stämme erfolgte im NRZ nach 


\begin{tabular}{|c|c|}
\hline Bundesland & $\begin{array}{l}\text { Zahl der Fälle } \\
\text { Erkr./Ausscheider }\end{array}$ \\
\hline Baden-Württemberg & 7 \\
\hline Bayern & 1 \\
\hline Berlin & 2 \\
\hline Bremen & 1 \\
\hline Hamburg & 2 \\
\hline Niedersachsen & 7 \\
\hline Nordrhein-Westfalen & 7 \\
\hline Rheinland-Pfalz & $4 / 1$ \\
\hline Sachsen & 4 \\
\hline Sachsen-Anhalt & $1 / 1$ \\
\hline Schleswig-Holstein & 3 \\
\hline Thüringen & 1 \\
\hline ohne Angabe & $-/ 1$ \\
\hline
\end{tabular}

den üblichen Standards [9] sowie zusätzlichen Kriterien [10]. Zur Unterscheidung von S. Paratyphi B und der Varietät S. Java wurde die Fermentation von d-Tartrat im Bleiazetat-Test geprüft [11]. Zur Feintypisierung der S. Paratyphi B-Stämme wurde die Lysotypie und die Plasmidcharakterisierung eingesetzt. Die Lysotypie der S. Paratyphi BStämme erfolgte als komplexe Typisierung nach der Methode von Felix und Callow sowie der Methode von Scholtens [12]. Die beiden Lysotypiebefunde werden durch einen Schrägstrich getrennt angegeben, z. B. Lysotyp (LT) Taunton/B7 bedeutet LT Taunton nach Felix und Callow und LT B7 nach Scholtens. Die Charakterisierung der Plasmide wurde als Bestimmung der Plasmidprofile nach der von Kado und Liu angegebenen Methode durchgeführt [13].

Im Ergebnis der durchgeführten Untersuchungen wurden für 36 Erkrankungsfälle, drei Ausscheider und zwei Kontakterkrankungen S. Paratyphi B, LT Taunton/B7 nachgewiesen. Alle diese Stämme waren plasmidfrei. Damit stimmten die Ergebnisse der Lysotypie in Deutschland mit denen der anderen acht europäischen Länder überein.

\section{Paratyphus B-Erkrankungen in anderen europäischen Ländern nach Türkeiaufenthalten}

Bei Urlaubsreisenden aus Norwegen (vier) und Schweden (zwei), die aus der Türkei zurückgekehrt waren, wurden im August 1999 Erkrankungen an Paratyphus B nachgewiesen. Als Erreger wurde S. Paratyphi B, LT Taunton ermittelt. Ein Austausch von Informationen zwischen den europäischen Ländern zeigte, dass auch Reisende anderer Länder nach Rückkehr aus der Türkei an Paratyphus B erkrankt waren. Die Mehrzahl der betroffenen Patienten war in Alanya und Umgebung gewesen. Die europaweiten Untersuchungen ergaben, dass von dem Paratyphusausbruch mindestens 309 Personen aus neun Ländern, vorwiegend Kinder und junge Erwachsene, betroffen waren. Da alle Erkrankungen durch einen einheitlichen Klon hervorgerufen worden waren, muss von einer gemeinsamen Infektionsquelle ausgegangen werden.

Die koordinierenden Arbeiten sowie Aktivitäten zur Untersuchung des Ausbruchs wurden durch das europäische Netzwerk Enter-net von London aus vorgenommen. Ende Oktober waren in insgesamt neun europäischen Ländern 309 Fälle an Paratyphus B bekannt [14]. Vorwiegend waren Kinder und junge Erwachsene hiervon betroffen. Molekularbiologische Untersuchungen in verschiedenen Ländern bestätigten, dass dem beobachteten Ausbruch von Erkrankungen an Paratyphus B nach Türkeireisen im Sommer 1999 ein Geschehen zugrunde lag, das durch einen einheitlichen Klon (Erreger mit gleichen Eigenschaften und Charakteristika) hervorgerufen worden war.

Eine internationale Expertengruppe war Anfang September (3. bis 14.9.1999) im Auftrag von Enter-net in Alanya, um vor Ort zur Klärung der Ursachen beizutragen. Eine multinationale Fall-Kontrollstudie wurde an $78 \mathrm{Er}$ krankten und 135 Kontrollpersonen durchgeführt. Hieran waren Dänemark, England und Wales, Finnland, Norwegen und Schweden beteiligt. Bisher liegen nur vorläufige Ergebnisse dieser Studie vor. Die zentrale Wasserversorgung sowie über den Handel bezogenes Wasser werden als Infektionsquelle ausgeschlossen. Hinweise auf Straßenhändler bzw. bestimmte Lebensmittel werden noch näher untersucht. Neben den in der vorliegenden Arbeit beschriebenen Erkrankungen an Paratyphus B in Deutschland traten weitere Fälle auch in Irland, den Niederlanden und der Schweiz auf. Eine Beteiligung Deutschlands an der internationalen Fall-Kontrollstudie wurde angestrebt, erwies sich jedoch als nicht realisierbar. Es gelang nicht, Kontrollpersonen, d.h. gesunde Personen, die zum gleichen Zeitpunkt in Alanya geweilt hatten, zu gewinnen und in die Studie einzubeziehen.

\section{Diskussion}

Angaben zu den Einzelfällen an Paratyphus in Deutschland liegen aus den Sondererhebungen des RKI erst seit 1997 vor. Bei der relativ geringen Zahl der jährlich auftretenden Fälle handelt es sich bei den bisher vorliegenden Daten insgesamt um nur kleine Fallzahlen. Diese lassen Auswertungen und Schlussfolgerungen daher nur in einem gewissen Umfang zu. Die Ergebnisse der Einzelfallerfassung bestätigen, dass viele der in Deutschland zur Meldung gelangten Erkrankungen an Paratyphus importiert wurden. Diese kamen in einer größeren Anzahl aus Ländern Asiens. Im Jahre 1999 führte ein Ausbruch von Paratyphus $B$ nach Türkeireisen im Vergleich zu früheren Jahren zu einer Erhöhung des Anteiles von Erkrankungen, die ihren Ursprung in der Türkei hatten. Obwohl es sich in Deutschland bei Paratyphus in den letzten Jahren um eine Erkrankung mit vergleichsweise wenigen Fällen handelte, stellt doch jede einzelne Erkrankung für den Betroffenen eine einschneidende persönliche Beeinträchtigung dar, die für einen großen Teil der Erkrankten mit einem Krankenhausaufenthalt verbunden ist (ca. 2/3 aller Erkrankten). Aber auch die Ausscheider sind für die Situation in Deutschland insgesamt von besonderer Bedeutung, da sie - ohne selbst zu erkranken - eine Gefahr für ihre Umgebung durch die Weiterverbreitung der Erreger darstellen. Deshalb gebührt der Verhinderung von Infektionen mit Paratyphus-Erregern und insbesondere ihrer Einschleppung aus dem Ausland verstärkte Beachtung [3].

In diesem Zusammenhang sollte die Aufmerksamkeit der Reisenden vor allem auf eine entsprechende Expositionsprophylaxe gelenkt werden. Dazu ge- 
hören das Vermeiden bestimmter Speisen, speziell außerhalb der Hotelanlagen, sowie die Verwendung von hygienisch einwandfreiem Wasser. Das heißt: keine rohen Salate und nur selbst geschältes Obst verzehren, Muscheln, Fisch und Fleisch nur ausreichend gegart $\mathrm{zu}$ sich nehmen; Trinkwasser abkochen oder Mineralwasser aus original verschlossenen Flaschen verwenden!

Ein beträchtlicher Teil der Erkrankungen tritt bei ausländischen Bürgern auf, die ihren Wohnsitz in Deutschland haben. Ein größerer Teil dieser Erkrankungen wird bei Besuchen im Heimatland, oft bei Verwandten, erworben. Einige Erkrankungen werden auch durch neu nach Deutschland einreisende Bürger importiert. Bei Bürgern ausländischer Herkunft werden aber auch Erkrankungen erfasst, die keinen direkten Bezug zu Reisen in andere Länder haben. Ob mit diesen Erkrankungsfällen Besucher aus den Heimatländern oder Ausscheider in der Familie oder näheren Umgebung in Zusammenhang gebracht werden können, muss in den meisten Fällen wohl offen bleiben. Anzumerken ist, dass sich die Ermittlungen der zuständigen Gesundheitsämter bei diesem Personenkreis meist sehr schwierig gestalten. Da relativ viele Erkrankungen an Paratyphus in Deutschland Bürger anderer Nationalitäten betreffen, erweisen sich die Möglichkeiten einer Einflussnahme auf das Verhalten in anderen Ländern und somit auf das Erwerben von Infektionen in diesen Ländern als eher gering und wenig erfolgversprechend.

Hinsichtlich der ermittelten Erregertypen kam es im betrachteten Zeitraum zu einem deutlichen Überwiegen von Paratyphus B im Vergleich zu Paratyphus A oder C. Damit erfolgte keine wesentliche Änderung dieser Verteilung im Vergleich zu früheren Zeiträumen [5]. Im Zusammenhang mit dem beschriebenen Ausbruch an Paratyphus B zeigen die Zahlen für 1999 (die zum gegenwärtigen Zeitpunkt noch vorläufig sind) eine noch deutlichere Dominanz von Paratyphus B. Notwendig wird es auch in Zukunft sein, über differenzierte Angaben zu den Erregertypen zu verfügen. Im vorliegenden Fall bedeutet das, dass eine Unterscheidung von Paratyphus $\mathrm{A}, \mathrm{B}$ und $\mathrm{C}$ gewährleistet sein muss. Die Datenerfassungssysteme, die im Zusammenhang mit dem Infektions- schutzgesetz zu etablieren sind, sollten dem Rechnung tragen und dies berücksichtigen.

Bei der Erfassung von Erkrankungen an Paratyphus kommt einer adäquaten Labordiagnostik eine große Rolle zu. Als ein Problem bei der Ermittlung der Paratyphus B-Erkrankungen nach Türkeireisen erwies sich, dass in einigen Fällen die Meldung im zuständigen Gesundheitsamt lediglich als "Salmonella, Gruppe B" vorlag, der weitere Befund, der auf Paratyphus hinwies, jedoch fehlte. Damit war eine Zuordnung der Erkrankung zu den Salmonellosen (Enteritis infectiosa) erfolgt. In vielen Fällen, in denen die Stämme zur Ausdifferenzierung weitergegeben wurden, konnten die Erkrankungen nachträglich als Paratyphus B eingeordnet werden. Wäre die bakteriologische Diagnostik jedoch nicht weitergeführt worden, so wären einige der Erkrankungen an $\mathrm{Pa}$ ratyphus $\mathrm{B}$ nicht als solche erkannt worden. Deshalb ist die Einschränkung der Diagnostik auf die Salmonellagruppe nicht ausreichend; zumindest sollte bei jedem Typhus- und Paratyphusverdacht der Serovar bestimmt werden. Wünschenswert ist es, zukünftig alle Stämme von Paratyphus-, aber auch Typhuserregern, dem NRZ zur Verfügung zu stellen, damit die dort etablierten Methoden zur Feindifferenzierung für alle in Deutschland gefundenen Stämme angewendet werden können und zu besseren Kenntnissen über die Situation in Deutschland bei diesen, vorwiegend aus dem Ausland mitgebrachten Erkrankungen, beitragen.

Durch die umfangreichen Aktivitäten, die im Zusammenhang mit der aktiven Fallfindung von Paratyphus B-Fällen nach Türkeireisen im Sommer 1999 standen, war es möglich, den größten Teil der Stämme einer Feintypisierung im NRZ zuzuführen. Das war die Grundlage für detaillierte Angaben zu den verursachenden Erregern. Es war gleichzeitig auch die Voraussetzung dafür, diese im internationalen Rahmen zu vergleichen. So zeigte sich, dass für alle Erreger, die in den verschiedenen Ländern untersucht worden waren, der Lysotyp Taunton nachgewiesen wurde. Alle untersuchten Stämme besaßen kein Plasmid.

\section{„Eine Verknüpfung von \\ Laborergebnissen und epidemiologischen Daten kann \\ zu besseren Erkenntnissen \\ über Herkunft und \\ Präventionsmöglichkeiten \\ führen."}

Im Laufe der Erfassung der Paratyphus B-Fälle nach Türkeireisen ergab sich eine enge Zusammenarbeit zwischen den Laboratorien, insbesondere dem NRZ und Mitarbeitern aus dem epidemiologischen Bereich des RKI, die über die Angaben zu den Einzelfällen verfügten bzw. nach Vorliegen des Typisierungsergebnisses die Ermittlungen zum Einzelfall aufnahmen. Deshalb konnten Laborergebnisse und epidemiologische Daten sehr gut miteinander verknüpft werden. Diese Zusammenarbeit sollte auch in Zukunft - zum gegenseitigen Nutzen fortgesetzt werden. Dies könnte zu vertieften Kenntnissen über die epidemiologische Situation sowohl bei Paratyphus als auch Typhus in Deutschland beitragen.

Insgesamt erwies sich die aktive Fallfindung zum Erfassen von Erkrankungen an Paratyphus B nach Türkeireisen im Sommer 1999 als gut geeignet, mit nur geringem Zeitverzug über detaillierte Informationen zu den Einzelfällen zu verfügen. Dadurch wurde die Mitarbeit Deutschlands im europäischen Netzwerk möglich. Nur auf diesem Wege gelang es, einen Großteil der Stämme an das NRZ schicken zu lassen, da diese in vielen Laboratorien in der Regel nicht für längere Zeit aufbewahrt werden und somit zu einem späteren Zeitpunkt für weitere Untersuchungen nicht mehr zur Verfügung gestanden hätten. Es muss jedoch eingeschätzt werden, dass diese Art der aktiven Erfassung von Einzelfällen nur ganz bestimmten Anlässen vorbehalten bleiben sollte, da sie einen hohen zusätzlichen Aufwand erfordert.

Alle im Rahmen der Fallfindung erhobenen epidemiologischen Daten wurden Enter-net, dem europäischen Surveillance-Netzwerk, zugeleitet und somit für eine Auswertung zugänglich gemacht. Im Gegensatz zu anderen europäischen Ländern erwies sich eine Teilnahme Deutschlands an der internationalen Fall-Kontrollstudie jedoch als 
nicht realisierbar. Es gelang nicht, mit Hilfe der Reiseveranstalter gesunde Kontrollpersonen, die sich zum gleichen Zeitpunkt in der Urlauberregion aufgehalten hatten, für die Studie zu rekrutieren [15]. Ein Reiseveranstalter hatte keinen Zugang zu den Personalien der Reisenden. Bei einem anderen Veranstalter blieb ein Schreiben des RKI an Alanyareisende ohne Rücklauf. Dieser misslungene Versuch, potentielle Kontaktpersonen zu rekrutieren, macht deutlich, dass eine Fall-Kontrollstudie bei Rückkehrern aus dem Ausland in Deutschland nahezu unmöglich ist, falls künftig nicht andere Wege zur Gewinnung von Kontrollpersonen für derartige Studien gefunden werden können.

An dieser Stelle soll allen an der aktiven Fallfindung Beteiligten - insbesondere den Mitarbeitern aus den obersten Landesbehörden, den Mitarbeitern der Gesundheitsämter, Krankenhäuser und Laboratorien - Dank für die vielfältigen Bemühungen und für die gute Zusammenarbeit ausgesprochen werden. Ebenso sei allen Mitarbeitern der Gesundheitsämter und Landesbehörden herzlich gedankt, die seit 1997 - durch Weitergabe der Angaben zu den Einzelfällen an Paratyphus anhand der Erhebungsbögen an das RKI zum Vorliegen der Informationen zum Auftreten von Paratyphus in der Bundesrepublik Deutschland ganz entscheidend beigetragen haben.

\section{Literatur}

1. Le Minor L (1988) Typing of Salmonella species. Eur J Clin Microbiol Infect Dis 7:214-218

2. Popoff MY, Bockemühl J,McWhorter-Murlin A (1992) Supplement 1991 (no.35) to the Kauffmann-White scheme. Res Microbiol 143:807-811

3. Brossmann D (1977) Zur Problematik der Ausscheider von Salmonella typhi und paratyphi B in hygienischer, epidemiologischer und soziologischer Hinsicht. Zbl Bakt Hyg, I Abt. Orig B 164:138-158

4. Dräger H (1971) Salmonellen, ihre Entstehung und Verhütung, 1. Auflage. Berlin: Akademie-Verlag; 99-208

5. Pöhn HP, Rasch G (1994) Statistik meldepflichtiger übertragbarer Krankheiten. BGA-Schriften 5/93. München:MMV Medizin Verlag, S32;132-134

6. Statistisches Bundesamt Gesundheitswesen, Fachserie 12, Reihe 2: Meldepflichtige Krankheiten (Wiesbaden 1990-1998)

7. Statistisches Bundesamt Gesundheitswesen, Fachserie 12, Reihe 4:Todesursachen (Wiesbaden 1990-1997)

8. Epidemiologisches Bulletin (1999) Hinweis auf importierte Paratyphus-und Typhuserkrankungen Epid Bull;35:262

9. Deutsche Gesellschaft für Hygiene und Mikrobiologie (Hrsg.). (1991) Verfahrensrichtlinien für die mikrobiologische Diagnostik, Isolierung und Identifizierung von Enterobacteriaceae, 6. Lieferung. Stuttgart, New York: Gustav Fischer Verlag, S 1-25

10. Old DC, Threlfall EJ (1998) Salmonella. In:Collier L, Ballows A, Sussman M (Hrsg.): Microbiology and Microbial Infections. Systematic Bacteriology. Chapter 41. New York: Oxford University Press, Vol. 2, S 969-996
11. Barker RM (1985) Utilization of d-tartaric acid by Salmonella paratyphi B and Salmonella java: comparison of anaerobic plate test, lead acetate test and turbidity test. J. Hyg.Camb. 95: 107-114

12. Rische H, ZieschÈ K (1973) Salmonella paratyphi B. In: Rische H (Hrsg.): Lysotypie und andere spezielle epidemiologische Laboratoriumsmethoden. Jena: Fischer Verlag, 1. Auflage: $565-86$

13. Kado Cl, Liu ST (1981) Rapid procedure for detection and isolation of large and small plasmids. J. Bacteriol. 145: 1365-1373

14. Eurosurveillance Weekly 1999 (28.10.1999) Case control study of Salmonella paratyphi B associated with travel to Alanya, Turkey: update.

15. Epidemiologisches Bulletin (1999) Paratyphus B nach Türkei-Reisen. Epid Bull; 45:333-334 